\title{
Culture-independent detection and characterisation of Mycobacterium tuberculosis and $M$. africanum in sputum samples using shotgun metagenomics on a benchtop sequencer
}

Tuberculosis remains a major global health problem. Laboratory diagnostic methods that allow effective, early detection of cases are central to management of tuberculosis in the individual patient and in the community. Since the 1880s, laboratory diagnosis of tuberculosis has relied primarily on microscopy and culture. However, microscopy fails to provide speciesor lineage-level identification and culture-based workflows for diagnosis of tuberculosis remain complex, expensive, slow, technically demanding and poorly able to handle mixed infections. We therefore explored the potential of shotgun metagenomics, sequencing of DNA from samples without culture or target-specific amplification or capture, to detect and characterise strains from the Mycobacterium tuberculosis complex in smear-positive sputum samples obtained from The Gambia in West Africa. Eight smear- and culture-positive sputum samples were investigated using a differential-lysis protocol followed by a kit-based DNA extraction method, with sequencing performed on a benchtop sequencing instrument, the Illumina MiSeq. The number of sequence reads in each sputum-derived metagenome ranged from 989,442 to $2,818,238$. The proportion of reads in each metagenome mapping against the human genome ranged from $20 \%$ to $99 \%$. We were able to detect sequences from the $M$. tuberculosis complex in all eight samples, with coverage of the H37Rv reference genome ranging from $0.002 \mathrm{X}$ to $0.7 \mathrm{X}$. By analysing the distribution of large sequence polymorphisms (deletions and the locations of the insertion element IS6110) and single nucleotide polymorphisms (SNPs), we were able to assign seven of eight metagenome-derived genomes to a species and lineage within the M. tuberculosis complex. Two metagenome- 
derived mycobacterial genomes were assigned to $M$. africanum, a species largely confined to West Africa; the others that could be assigned belonged to lineages T, H or LAM within the clade of "modern" M. tuberculosis strains. We have provided proof of principle that shotgun metagenomics can be used to detect and characterise $M$. tuberculosis sequences from sputum samples without culture or target-specific amplification or capture, using an accessible benchtop-sequencing platform, the Illumina MiSeq, and relatively simple DNA extraction, sequencing and bioinformatics protocols. In our hands, sputum metagenomics does not yet deliver sufficient depth of coverage to allow sequence-based sensitivity testing; it remains to be determined whether improvements in DNA extraction protocols alone can deliver this or whether culture, capture or amplification steps will be required. Nonetheless, we can foresee a tipping point when a unified automated metagenomics-based workflow might start to compete with the plethora of methods currently in use in the diagnostic microbiology laboratory. 
2 Culture-independent detection and characterisation of

3 Mycobacterium tuberculosis and M. africanum in

4 sputum samples using shotgun metagenomics on a

5 benchtop sequencer

\author{
6 Emma L. Doughty ${ }^{1}$ \\ $7 \quad$ Martin J. Sergeant ${ }^{1}$ \\ 8 Ifedayo Adetifa ${ }^{2}$ \\ 9 Martin Antonio ${ }^{1,2}$ \\ 10 Mark J. Pallen ${ }^{1}$ \\ 111 Microbiology and Infection Unit, Warwick Medical School, University of \\ 12 Warwick, Gibbet Hill Road, Coventry, United Kingdom, CV4 7AL \\ 132 Medical Research Council Unit, Fajara, The Gambia \\ 14 *for correspondence: email m.pallen@warwick.ac.uk
}




\section{Introduction}

16 Tuberculosis (TB) is an infection, primarily of the lungs, caused by Mycobacterium tuberculosis and related species within the $M$. tuberculosis complex. TB remains a major global health problem, second only to HIV/AIDS in terms of global deaths from a single infectious agentaccording to estimates from the World Health Organisation (WHO), 8.6 million people developed TB in 2012 and 1.3 million died from the disease, including 320,000 deaths among HIV-positive individuals (WHO, 2013).

Central to management of TB in the individual patient and in the community are laboratory diagnostic methods that allow effective, early detection of cases. Since the pioneering work of Koch and Ehrlich in the 1880s, laboratory diagnosis of pulmonary TB has largely relied on acidfast staining of sputum samples and culture on selective laboratory media for the isolation of mycobacteria (Ehrlich, 1882; Koch, 1882). Microscopy is still generally used as a first-line diagnostic approach and as the only laboratory approach in resource-poor settings (Drobniewski et al., 2012) Smear-positivity is also used as a guide to infectivity and responsiveness to treatment. However, microscopy fails to provide species-level identification of acid-fast bacilli (Maiga et al., 2012). Such identification is important in guiding treatment, because pathogenic mycobacteria from outside the $M$. tuberculosis complex often fail to respond to conventional anti-TB treatment (Maiga et al., 2012). Furthermore, there are important differences in response to treatment even within the $M$. tuberculosis complex. M. bovis and $M$. canettii fail to respond to the first-line anti-tuberculous agent pyrazinamide_-as a result, failure to recognise $M$. bovis as a cause of TB can have fatal consequences (Allix-Beguec et al., 2010). In addition, M. canettii appears to show decreased susceptibility to a promising new anti-TB drug candidate, PA-824 (Feuerriegel et al., 2011; Feuerriegel et al., 2013).

There is also increasing recognition of lineage- or species-specific differences in pathogen biology within the $M$. tuberculosis complex. M. africanum, which is largely restricted to West Africa, where it causes up to half of human pulmonary TB, is associated with less transmissible and less severe infection than typical strains of the "modern" $M$. tuberculosis clade (de Jong et al., 2010b). Similarly, M. canettii, restricted to the horn of Africa, and M. bovis, both usually a spillover from animals, transmit relatively poorly from human to human (Fabre et al., 2010; Gonzalo-Asensio et al., 2014). By contrast, the Beijing-W lineage of M. tuberculosis sensu stricto, which has spread around the world in recent decades, appears to cause more aggressive disease and is more likely to become drug-resistant (Nicol and Wilkinson, 2008; Borgdorff and van Soolingen, 2013).

Owing to the slow growth rate of the $M$. tuberculosis complex, traditional culture-based diagnosis of TB typically takes several weeks or even months. Similarly, conventional phenotypic mycobacterial sensitivity testing remains slow and may not be reliable for all classes of antituberculous agent. In recent decades, automated detection of growth in liquid culture, through e.g. the mycobacteria growth indicator tube (MGIT), has led to improvements in the speed and ease of diagnosis, so that diagnosis by culture is now often possible within a fortnight (Pfyffer et al., 1997).

55 However, in comparison to most other laboratory procedures, culture-based diagnostic workflows 56 for TB remain complex, expensive, slow, technically demanding and require expensive 
biocontainment facilities. Furthermore, as isolation of mycobacteria in pure culture and sensitivity testing remain onerous, in resource-poor settings these steps are omitted and, even in well-resourced laboratories, typically only one or a few single-colony subcultures are followed up from each sample. This leads to under-recognition of mixed infections, where more than one strain from the M. tuberculosis complex is present or where TB co-occurs with infection by other mycobacteria (Shamputa et al., 2004; Warren et al., 2004; Cohen et al., 2011; Wang et al., 2011). This can lead to difficulties in treatment when strains or species susceptible to conventional antituberculous treatment co-exist with resistant strains or species within the same patient (HingleyWilson et al., 2013).

As an alternative to culture and phenotypic sensitivity testing, the WHO has recently recommended a new, rapid, automated, real-time amplification-based TB diagnostic test, the Xpert MTB/RIF assay (WHO, 2011). This system allows simultaneous detection of $M$. tuberculosis and rifampicin-resistance mutations in a closed system, suitable for use in a simple laboratory setting, while providing a result in less than two hours directly from sputum samples (Helb et al., 2010). However, this approach performs suboptimally on mixed infections, fails to provide the full range of clinically relevant information (e.g. speciation, susceptibility to other agents) and, in sampling only a small fraction of the genome, affords no insight into pathogen biology, evolution, and epidemiology (Zetola et al., 2014).

Epidemiological investigation of clinical isolates from the $M$. tuberculosis complex plays an important role in the management and control of TB. A range of molecular typing schemes have been developed, including IS6110 fingerprinting, mycobacterial interspersed repetitive unitvariable number of tandem repeat (MIRU-VNTR) and spoligotyping (Jagielski et al., 2014). These approaches can be valuable in distinguishing relapse from re-infection and in recognising mixed infections within the individual patient, as well as identifying sources of infection, detecting outbreaks and tracking spread of lineages within a community. However, as these approaches usually require isolation of the pathogen in pure culture, clinically relevant typing data is typically not available until 1-2 months after collection of a sputum sample.

Over the past fifteen years, whole-genome sequencing has been applied to a steadily wider range of isolates from M. tuberculosis and related species (Cole et al., 1998; Brosch et al., 2002; Gutierrez et al., 2009). These efforts have shed light on the evolution and population structure of this group of pathogens, showing that members of the M. tuberculosis complex are reproductively isolated, engaging in almost no horizontal gene transfer and showing a clonal population structure in which lineages diverge through a limited set of genetic changes, including point mutations, deletions, movement of insertion elements and rearrangements within repetitive regions. Whole-genome analyses allow isolates to be assigned to a range of species, global lineages and sub-lineages on the basis of single nucleotide polymorphisms (SNPs) and large sequence polymorphisms (typically deletions, which are often termed "regions of difference" or RDs, and insertion of the transposable element IS6110).

In recent years, the availability of rapid, cheap high-throughput sequencing and, particularly, the arrival of user-friendly benchtop sequencing platforms, such as the Illumina MiSeq (Loman et al., 2012a; Loman et al., 2012b), have led to the widespread use of whole-genome sequencing in TB sensitivity testing and epidemiology, with adoption of whole-genome sequencing for routine use in some TB reference laboratories (Gardy et al., 2011; Koser et al., 2012; Roetzer et al., 2013; 
100 Walker et al., 2013; Walker et al., 2014). However, high-throughput sequencing has not yet been

101 used as a diagnostic tool for TB, because it has been assumed that one needs to subject clinical

102 samples to prolonged culture before sufficient mycobacterial DNA can be obtained for whole-

103 genome sequencing and analysis. Some researchers (Koser et al., 2013) have recently challenged

104 this assumption by obtaining mycobacterial genome sequences from DNA extracted directly from

105 a three-day MGIT culture of a sputum sample. However, this begs the questions: why bother with

106 culture; why not obtain mycobacterial genome sequences directly from a sputum sample, without

107 culture?

108 Shotgun metagenomics - that is the unbiased sequencing en masse of DNA extracted from a 109 sample without target-specific amplification or capture-has provided a powerful assumption-

110 free approach to the recovery of bacterial pathogen genomes from contemporary and historical 111 material (Pallen, 2014). This approach allowed an outbreak strain genome to be reconstructed 112 from stool samples from the 2011 Escherichia coli O104:H4 outbreak and has proven successful 113 in obtaining genome-wide sequence data for Borrelia burgdorferi, M. leprae, M. tuberculosis and

114 Brucella melitensis from long-dead human remains (Keller et al., 2012; Chan et al., 2013; Loman 115 et al., 2013; Schuenemann et al., 2013; Kay et al., 2014). Metagenomics has recently provided 116 clinically useful information in cases of chlamydial pneumonia and neuroleptospirosis (Fischer et 117 al., 2014; Wilson et al., 2014).

118 Here, we explore the potential of metagenomics in detecting and characterising Mycobacterium 119 tuberculosis and M. africanum strains in smear-positive sputum samples from patients from The 120 Gambia in West Africa. 


\section{Microbiological analysis and sample selection}

123 Eight smear- and culture-positive sputum samples were selected for metagenomic analysis from

124 specimens collected in May 2014 under the auspices of the Enhanced Case Finding project

125 (http://clinicaltrials.gov/show/NCT01660646). The joint Gambia Government/MRC Ethics

126 Committee approved this investigation under reference SCC 1232 and informed written consent

127 was obtained for all participants. The sputum samples were collected by expectoration into a

128 sterile cup and transported on ice to the TB laboratory at the MRC Gambia unit within 24 hours

129 of collection.

130 Prior to selection for metagenomic investigation, an aliquot of each sample was subjected to microbiological analysis. These specimens were decontaminated by the sodium hydroxide and $N$ acetyl-l-cysteine (NaOH/NALC) method, with final concentrations of $1 \%$ for $\mathrm{NaOH}, 1.45 \%$ sodium citrate and $0.25 \%$ for NALC. Sputum smears were prepared by centrifuging 3-10 ml decontaminated sputum and then resuspending pellets in $2 \mathrm{ml}$ buffer. Smears were stained with auramine-O and then examined by fluorescence microscopy. Positive smears were confirmed by Ziehl-Neelsen staining. 20-100 fields were examined at 1000X magnification and smear-positive samples were scored quantitatively as $1+, 2+$ or $3+$ (Kent and Kubica 1985). The presence of $M$. tuberculosis complex in samples was confirmed by culture in the BACTEC MGIT 960 Mycobacterial Detection System and on slopes of Löwenstein-Jensen medium. Cultured isolates were subjected to spoligotyping as previously described (Kamerbeek et al., 1997; de Jong et al., 2009).

\section{2 \\ DNA extraction using differential lysis}

143 DNA extraction was performed in the TB laboratory in the MRC Unit in The Gambia. Aliquots

144 of unprocessed sputum were subjected to a differential lysis protocol, modified from a published 145 method for metagenomic analysis of sputum from cystic fibrosis patients (Lim et al., 2012). In 146 this method, human cells are subjected to osmotic lysis and then the liberated human DNA is 147 removed by DNase treatment. To monitor contamination within the laboratory, we processed two 148 negative-control samples containing only sterile water via the same method.

149 At the start of the differential lysis protocol, a $1 \mathrm{~mL}$ aliquot of whole sputum was mixed with 1 $150 \mathrm{~mL}$ decongestant solution $(0.25 \mathrm{~g} \mathrm{~N}$-acetyl L-cysteine, $25 \mathrm{~mL} 2.9 \%$ sodium citrate, $25 \mathrm{~mL}$ water $)$ until liquefied and incubated for $15 \mathrm{~min}$ at room temperature. $48 \mathrm{~mL}$ phosphate-buffered solution $(\mathrm{pH} 7)$ was added and mixed thoroughly, before centrifugation at $3220 \mathrm{x} \mathrm{g}$ for $20 \mathrm{~min}$. The pellet was resuspended in $10 \mathrm{~mL}$ sterile deionised water and incubated at room temperature for $15 \mathrm{~min}$, so that human cells undergo osmotic lysis, while mycobacterial cells remain intact. The centrifugation and resuspension-in-water steps were repeated before a final round of centrifugation. The pellet was then treated with the RNase-Free DNase Set (Qiagen), adding 25 $\mu \mathrm{L}$ DNase I (2.73 Kunitz units per $\mu \mathrm{L}), 100 \mu \mathrm{L}$ RDD buffer and $875 \mu \mathrm{L}$ sterile water. The sample was then incubated at room temperature for 2 hours, with repeated inversion of the tubes. The sample underwent two rounds of centrifugation and resuspension of the pellet in $10 \mathrm{~mL}$ TE buffer

160 (0.01M Tris-HCl, 0.001 M EDTA, pH 8.0). Finally, before DNA extraction began, the sample 161 was centrifuged and the pellet was resuspended in $500 \mu \mathrm{L}$ TE buffer. On completion of the 
162 differential lysis protocol, samples underwent heat treatment at $75^{\circ} \mathrm{C}$ for $10 \mathrm{~min}$, followed by

163 DNA extraction using a commercial kit, the NucleoSpin Tissue-Kit (Macherey-Nagel, Duren,

164 Germany), according to the manufacturer's protocol for hard-to-lyse bacteria.

\section{Library preparation and sequencing}

166 DNA samples were sent to Warwick Medical School, Coventry, UK, where all further laboratory

167 and bioinformatics analyses were performed. The concentration of DNA present in each extract

168 was determined using the Qubit 2.0 fluorometer and Qubit@ dsDNA Assay Kits according to the

169 manufacturer's protocol (Invitrogen Ltd., Paisley, United Kingdom), using the HS (high-

170 sensitivity) or BR (broad-range) kits, depending on the DNA concentration. There was no

171 detectable DNA in the negative control samples with the HS kit, which is sensitive down to 10

$172 \mathrm{pg} / \mu \mathrm{L}$. DNA extracts were diluted to $0.2 \mathrm{ng} / \mu \mathrm{L}$ and were then converted into sequencing

173 libraries, using the Illumina Nextera XT sample preparation kit according to the manufacturer's

174 instructions (Illumina UK, Little Chesterford, United Kingdom). The libraries were sequenced on

175 the Illumina MiSeq at the University of Warwick.

Identification of human and mycobacterial sequences

177 Sequence reads were mapped against the genome of Mycobacterium tuberculosis H37Rv

178 (GenBank accession numbers AL123456) and the human reference genome hg19 (GenBank

179 Assembly ID: GCA_000001405.1), using Bowtie2 version 2.1.0 (Langmead and Salzberg, 2012),

180 using relaxed and stringent protocols. The relaxed protocol exploited the option - - very -

181 sensitive-local. The stringent protocol allowed only limited mismatches (3 per 100 base

182 pairs) and soft clipping of poor quality ends, by exploiting the options - - ignore - quals

183 --mp 10,10 --score-min L, 0,0.725 --1ocal --ma 1. A custom-built script was

184 used to convert coverage data from the BAM files into a tab-delineated format that was then

185 entered into Microsoft Excel, which was then used to generate coverage plots. Metagenomic

186 sequence reads from this study (excluding those that mapped to the human genome) have been

187 deposited in the European Nucleotide Archive (project accession number pending).

\section{Species and lineage assignment using low-coverage SNPs}

189 For the phylogenetic analysis using SNPs, we selected representative genomes from each of the

190 species and major lineages within the M. tuberculosis complex that infect humans, drawing on

191 lineage designations reported by PolyTB. Genome sequences were taken from entries in the short

192 read archive ERP000276 and ERP000124 (http://www.ncbi.nlm.nih.gov/Traces/sra/). We then

193 mapped these genomes against M. tuberculosis H37Rv with Bowtie2 under default settings and

194 then called SNPs using VarScan2 (Koboldt et al., 2012). Any SNPs that fell within a set of

195 previously published repetitive genes were excluded from further analysis (Comas et al., 2010).

196 SNPs were used to construct a tree with RAxML version 7 (Stamatakis, 2014), using default

197 parameters with the GTR-gamma model. Reads from the metagenome from each sample were

198 mapped against the reference strain M. tuberculosis H37Rv using the default settings in Bowtie2

199 and the majority base called from each SNP position with no quality filtering. If no base was

200 present at the position, a gap was used. The pplacer suite of programs (Matsen et al., 2010) was

201 then used to assign the sequence to a species and lineage on the mycobacterial tree. 


\section{Lineage assignment using IS6110-insertion-site profiles}

203 We mapped each metagenome against the sequence of IS6110 (Genbank accession number: 204 AJ242908) using Bowtie's - - local option, which performs a softclipping of the mapped

205 sequences. We then extracted IS6110-flanking sequences by retrieving all sequences $>30 \mathrm{bp}$ that 206 had that had been softclipped from the ends of the element. These sequences were then mapped 207 against the H37Rv genome using Bowtie2 and the coordinates of the IS6110 insertion points 208 determined.

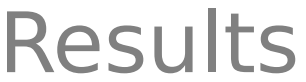

\section{Detection of the M. tuberculosis complex in sputum samples}

212 We obtained metagenomic sequences from eight smear- and culture-positive sputum samples.

213 The number of sequence reads in each sputum-derived metagenome ranged from 989,442 to

$2142,818,238$ (Table 1). The proportion of reads from each sample mapping to the human reference

215 genome hg19 varied from $20 \%$ to $99 \%$.

216 Coverage from reads mapping to the genome of the $M$. tuberculosis reference strain $\mathrm{H} 37 \mathrm{Rv}$ under 217 relaxed settings ranged from $0.009 \mathrm{X}$ to $1.3 \mathrm{X}$ (Table 2). However, we suspected that many of the 218 matches represented false-positives. To confirm our suspicion, we calculated the average read 219 depth at the positions where reads matched.

220 If the matches occurred because of sequence identity with conserved genes from other species, one would expect there to be multiple reads matching each mapped position, whereas for a shotgun library where the coverage is less than $1 \mathrm{X}$, one would expect the average read depth to be around 1 . However, as we created our sequence libraries using a paired-end protocol, there will be variable overlap between reads originating from the same DNA fragment, so one would expect the average read depth for a genuine random shotgun under these conditions to sit between 1 and 2. However, when mapping was performed under relaxed conditions, the average read depth was $>2$ in six of the eight samples and in two cases was $>7$ (Table 2), indicating a major contribution from spurious matches to conserved genes.

To restrict matches to the $\mathrm{H} 37 \mathrm{Rv}$ genome to genuine on-target alignments, we then mapped each metagenome against the reference strain under high-stringency conditions $(\leq 3$ mismatches per 100 base pairs, with soft clipping of poor quality ends). This led to a decrease in reads mapping to H37Rv in all samples, with coverage of the H37Rv under stringent settings ranging from

$2330.002 \mathrm{X}$ to $0.7 \mathrm{X}$. Nonetheless, we recovered between $\sim 11,000$ and 3 million base pairs of $M$.

234 tuberculosis sequence from our samples under such stringent conditions (Table 2). The average

235 read depth in the samples fell to between 1.2 and 1.9, consistent with expectations for a random

236 shotgun (Table 2).

237 Phylogenetic placement of M. tuberculosis strains using 238 SNPs

239 Conventional phylogenetic methods based on identification of trusted SNPs cannot be applied to 240 the kinds of low-coverage genome sequences we have obtained here. However, the technique of 241 "phylogenetic placement" provides an alternative solution (Matsen et al., 2010; Kay et al., 2014). 
242 Here, one draws on a fixed reference tree, computed from high-coverage genomes, and places the

243 unknown query sequence on to the tree using programs such as pplacer (Matsen et al., 2010). To perform phylogenetic placements on our samples, we derived a set of phylogenetically informative SNPs from representatives of the major lineages within the M. tuberculosis complex. We then analysed reads from each of the sputum metagenomes that aligned to equivalent positions in the H37Rv genome.

248 Using this approach, despite the low coverage, we could confidently assign (with a posterior probability of $>0.97$ ), all but one of the metagenome-derived mycobacterial genomes to a species and lineage within the $M$. tuberculosis complex (Figure 1). In all these cases, the conclusions from metagenomics matched those from spoligotyping of cultured isolates (Table 3). For two of the samples (K3, K5), the metagenome-derived genome was assigned to $M$. africanum clade 2, which is consistent with the known high-prevalence of this lineage in The Gambia (de Jong et al., 2010a). Five samples were assigned to the Euro-American lineage (also termed Lineage 4), which sits within the clade of modern M. tuberculosis strains and which is known to be highly prevalent in The Gambia (de Jong et al., 2010a). Phylogenetic placement allowed three of these samples to be assigned to sub-lineage $\mathrm{H}$, one to the T-clade and one to the LAM clade.

\section{Species and lineage assignment using IS6110 insertion} sites

From four samples, we were able to retrieve information on IS6110 insertion sites (Table 4). In two of the three samples (K2, K4) assigned to the H clade by phylogenetic placement, we discovered IS6110 insertion sites that had previously been reported as specific to the Haarlem or H clade (HSI1, HSI2, HSI3), thereby confirming the SNP-based lineage assignment (CubillosRuiz et al., 2010). In the sample assigned to the LAM clade, we retrieved information on a single IS6110 insertion site, which disrupts the coding sequence Rv3113. This insertion has been reported as specific to the LAM clade (Lanzas et al., 2013), again confirming the SNP-based lineage assignment. In one of the two samples assigned to M. africanum, we retrieved information on a single IS6110 insertion site. However, this insertion appeared to be absent from all other available genome-sequenced strains from the M. tuberculosis complex, so was phylogenetically uninformative.

Here, we have provided proof of principle that shotgun metagenomics can be used to detect and characterise $M$. tuberculosis sequences from sputum samples without culture or target-specific amplification or capture, using an accessible benchtop-sequencing platform, the Illumina MiSeq, and relatively simple DNA extraction, sequencing and bioinformatics protocols.

There are several proven or potential advantages to metagenomics as a diagnostic approach for pulmonary TB. By circumventing the need for culture, it could provide information more quickly than conventional approaches. Even in this proof-of-principle study, for most samples it has provided more detailed information than conventional approaches, including spoligotyping. In addition, it represents an open-ended one-size-fits-all approach that could allow the reunification

281 of TB microbiology with other sputum microbiology, particularly as metagenomics has already

282 been shown to work on other respiratory tract pathogens, including bacteria and viruses (Lysholm et al., 2012; Fischer et al., 2014). It also aids in the detection of mixed infections (Chan et al., 
284 2013; Koser et al., 2013), which are clinically important, but hard to recognise (Shamputa et al.,

285 2004; Warren et al., 2004; Cohen et al., 2011; Wang et al., 2011; Hingley-Wilson et al., 2013).

286 However, as things stand, there are several important limitations to metagenomics as a diagnostic 287 approach. Our study has been limited to the investigation of smear-positive sputum samples, 288 where a diagnosis can already be obtained quickly and easily by microscopy; considerable 289 improvements in sensitivity are likely to be needed before metagenomics can be made to work on 290 smear-negative culture-positive samples. However, it is worth stressing that smear-positive cases are the most important TB cases in terms of infectivity and severity of disease and rapid, accurate diagnosis and epidemiological investigation of such samples is likely to aid TB control (Shaw and Wynn-Williams, 1954; Colebunders and Bastian, 2000; Wang et al., 2008). Plus, for all our samples, metagenomics goes beyond mere detection of acid-fast bacilli to deliver clinically important information at the level of species and lineage within the M. tuberculosis complex.

Surprisingly, metagenomics has not proven quite so informative when applied to contemporary sputum samples as when applied to historical samples, from which we have gained much higher coverage of pathogen genomes, which allowed recognition of phylogenetically informative large sequence polymorphisms (Chan et al., 2013; Kay et al., 2014). Furthermore, in our hands, sputum metagenomics does not yet deliver sufficient depth of coverage of TB genomes to allow the accurate SNP calling necessary for sequence-based sensitivity testing. It remains unclear whether increased depth of coverage can be achieved by refinements in DNA extraction protocols aloneor whether one might need to sacrifice the speed, simplicity and open-endedness of shotgun metagenomics by incorporating amplification of mycobacterial DNA or cells (i.e. by culture in MGIT tubes (Koser et al., 2013)) or by capture of mycobacterial cells or DNA (Sweeney et al., 2006; Bouwman et al., 2012; Schuenemann et al., 2013).

Some have argued that metagenomics is too expensive for routine use (Köser et al., 2014).

308 However, the same was true of whole-genome sequencing a few years ago; in this study, reagent costs amounted to $<£ 50$ per sample. Plus, with minor modifications, we anticipate that DNA extraction could be completed in a few hours of receipt of a sputum sample and sequencing and analysis within a few days. In addition, now that cultured TB isolates are being routinely genome sequenced in many laboratories (Koser et al., 2012; Kohl et al., 2014), a catalogue of local TB genomes will be available for comparison with the metagenome-derived genomes, facilitating epidemiological analyses

315 With likely future improvements in the ease, throughput and cost-effectiveness of sequencing,

316 twinned with commoditisation of laboratory and informatics workflows, one can foresee a tipping 317 point when a unified automated metagenomics-based workflow might start to compete with the 318 plethora of methods currently in use in the diagnostic microbiology laboratory, while also 319 delivering additional useful information on epidemiology, antimicrobial resistance and pathogen 320 biology.

322 We thank Catherine Okoi for providing an introduction to the MRC Gambia TB laboratory,

323 Abigail Ayorinde for spoligotype analysis and Ousman Secka for sending the DNA extracts to

324 Warwick from The Gambia. We are grateful to the TB field team lead by Francis Oko and the 
325 Mycobacteriology team at MRC Unit The Gambia. We thank Chrystala Constantinidou, Gemma 326 Kay and Andrew Millard for advice on laboratory and bioinformatics procedures. 
327 Tables

328 Table 1 Sample characteristics and sequencing results

\begin{tabular}{|r|r|r|r|r|}
\hline Sample & \multicolumn{1}{l|}{$\begin{array}{l}\text { ZN } \\
\text { grade }\end{array}$} & $\begin{array}{l}\text { DNA concentration } \\
\text { in extract }(\boldsymbol{\mu g} / \mathbf{m L})\end{array}$ & $\begin{array}{l}\text { Total no. reads } \\
\text { aligning to } \\
\text { human genome }\end{array}$ \\
\hline $\mathrm{K} 1$ & $3+$ & 27.8 & 989,442 & 73.71 \\
\hline $\mathrm{K} 2$ & $3+$ & 2.28 & $2,170,640$ & 78.46 \\
\hline $\mathrm{K} 3$ & $2+$ & 71 & $1,617,808$ & 99.3 \\
\hline $\mathrm{K} 4$ & $2+$ & 250 & $1,204,408$ & 97.22 \\
\hline $\mathrm{K} 5$ & $2+$ & 7.7 & $1,537,676$ & 74.17 \\
\hline $\mathrm{K} 6$ & $2+$ & 48.8 & $2,411,708$ & 97.47 \\
\hline $\mathrm{K} 7$ & $1+$ & 25 & $2,818,238$ & 50.59 \\
\hline $\mathrm{K} 8$ & $1+$ & 0.63 & $1,851,892$ & 20.29 \\
\hline
\end{tabular}

329 Table 2 Mapping to $M$. tuberculosis $\mathbf{H 3 7 R v}$ reference genome

\begin{tabular}{|r|r|r|r|r|r|r|}
\hline Sample & \multicolumn{2}{|c|}{ Under relaxed mapping conditions } & \multicolumn{2}{|c|}{ Under stringent mapping conditions } \\
\cline { 2 - 7 } & $\begin{array}{r}\text { Bases } \\
\text { aligning } \\
\text { to H37Rv }\end{array}$ & $\begin{array}{r}\text { Coverag } \\
\text { e of } \\
\text { H37Rv }\end{array}$ & $\begin{array}{r}\text { Average } \\
\text { read } \\
\text { depth }\end{array}$ & $\begin{array}{r}\text { Bases } \\
\text { aligning } \\
\text { to H37Rv }\end{array}$ & $\begin{array}{r}\text { Coverage } \\
\text { of H37Rv }\end{array}$ & $\begin{array}{r}\text { Average } \\
\text { read depth }\end{array}$ \\
\hline K1 & 410,228 & 0.093 & 2.2 & 141,906 & 0.032 & 1.3 \\
\hline K2 & $5,685,901$ & 1.289 & 2.3 & $3,057,187$ & 0.693 & 1.9 \\
\hline K3 & 99,643 & 0.023 & 1.3 & 54,413 & 0.012 & 1.2 \\
\hline K4 & 40,019 & 0.009 & 1.9 & 10,840 & 0.002 & 1.3 \\
\hline K5 & 732,623 & 0.166 & 2.5 & 238,451 & 0.054 & 1.3 \\
\hline K6 & 94,023 & 0.021 & 2.3 & 34,704 & 0.008 & 1.7 \\
\hline K7 & $1,366,309$ & 0.310 & 11.4 & 50,873 & 0.012 & 1.5 \\
\hline K8 & $1,725,816$ & 0.391 & 7.7 & 109,514 & 0.025 & 1.3 \\
\hline
\end{tabular}


Table 3 Species and lineage assignments by phylogenetic placement and spoligotyping

\begin{tabular}{|c|c|c|c|c|}
\hline \multirow[t]{2}{*}{ Sample } & \multicolumn{2}{|c|}{ Phylogenetic placement by pplacer } & \multicolumn{2}{|r|}{ Spoligotyping } \\
\hline & Species, lineage, clade & $\begin{array}{l}\text { Posterior } \\
\text { probability }\end{array}$ & Lineage & Spoligotype \\
\hline $\begin{array}{l}\mathrm{K} 1 \\
+ \\
+0 \\
\end{array}$ & $\begin{array}{l}\text { M. tuberculosis } \\
\text { Euro-American / } \\
\text { Lineage } 4 \\
\text { LAM clade }\end{array}$ & 1 & Euro-American & 1101111111110111111100001111111100001111011 \\
\hline $\begin{array}{l}\overline{\mathrm{K} 2} \\
\frac{\mathrm{D}}{2} \\
\overline{\mathrm{D}} \\
\end{array}$ & $\begin{array}{l}\text { M. tuberculosis } \\
\text { Euro-American / } \\
\text { Lineage } 4 \\
\text { H clade }\end{array}$ & 1 & Euro-American & 1111111111111111111111111111110100001111111 \\
\hline 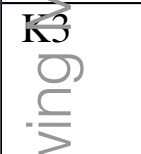 & $\begin{array}{l}\text { M. africanum } \\
\text { Lineage } 6 \\
\text { M. africanum clade } 2\end{array}$ & 1 & West African 2 & 1111110001111111111000001000011111111101111 \\
\hline 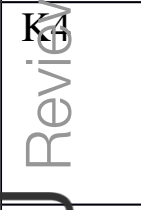 & $\begin{array}{l}\text { M. tuberculosis } \\
\text { Euro-American / } \\
\text { Lineage } 4 \\
\text { H clade }\end{array}$ & 0.99 & Euro-American & 1111111111111111111111111111110100001111111 \\
\hline $\mathrm{Ka}$ & $\begin{array}{l}\text { M. africanum } \\
\text { Lineage } 6 \\
\text { M. africanum clade } 2\end{array}$ & 1 & West African 2 & 1111110001111111111111111111111111111101111 \\
\hline (4) & Not determined & & West African 2 & 1111110001111111111111111111111111111101111 \\
\hline 57 & $\begin{array}{l}\text { M. tuberculosis } \\
\text { Euro-American / } \\
\text { Lineage } 4 \\
\text { H clade }\end{array}$ & 0.97 & Euro-American & 1111111111111111111111111111110100001111111 \\
\hline K8 & $\begin{array}{l}\text { M. tuberculosis } \\
\text { Euro-American / } \\
\text { Lineage } 4 \\
\text { T clade }\end{array}$ & 1 & Euro-American & 1111110000000000000000000111111100001111111 \\
\hline
\end{tabular}


331 Table 4 IS6110 profiles

\begin{tabular}{|c|c|c|c|c|}
\hline Sample & $\begin{array}{l}\text { No. reads } \\
\text { mapping to } \\
\text { IS6110 }\end{array}$ & $\begin{array}{l}\text { No. reads } \\
\text { spanning IS6110 } \\
\text { insertion site }\end{array}$ & IS6110 insertion site coordinates & Comments \\
\hline K1 & 11 & 1 & 3480371 & Specific to LAM clade \\
\hline 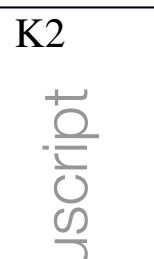 & 199 & 22 & $\begin{array}{l}2610861 \text { (HSI1), 1075947-1075950 } \\
\text { (HSI2), 1715974 (HSI3). } \\
212132-212135,483295-483298, \\
888787,1695606,1986622-1986625, \\
3120523\end{array}$ & $\begin{array}{l}\text { HSI1, HSI2, HSI3 } \\
\text { specific to H clade: }\end{array}$ \\
\hline $\mathrm{K} 3$ & 2 & 0 & Not determined & \\
\hline $\mathrm{K} \stackrel{1}{\mathbf{0}}$ & 6 & 2 & 2610861-2610864 (HSI1) & HSI1 specific to $\mathrm{H}$ clade \\
\hline $\mathrm{K5}^{2}$ & 4 & 1 & 2631765 & Unique so uninformative \\
\hline $\mathrm{K} 6$ & 0 & 0 & Not determined & \\
\hline$K \bar{Z}$ & 2 & 0 & Not determined & \\
\hline $\mathrm{K} 80$ & 5 & 0 & Not determined & \\
\hline
\end{tabular}




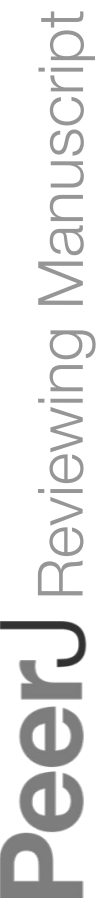

PeerJ reviewing PDF | (v2014:07:2463:1:0:CHECK 28 Aug 2014) 


\section{${ }_{332}$ Supplementary Data}

333 Detailed phylogenetic placement of metagenome-derived genomes

334 SNP matrix used to generate tree.

335 List of repetitive genes excluded from SNP calling. 
337 Allix-Beguec, C, M Fauville-Dufaux, K Stoffels, D Ommeslag, K Walravens, C Saegerman, and 338 P Supply. 2010. Importance of identifying Mycobacterium bovis as a causative agent of human

339 tuberculosis. Eur Respir J 35, no. 3: 692-694.

340 Borgdorff, MW, and D van Soolingen. 2013. The re-emergence of tuberculosis: what have we learnt from molecular epidemiology? Clin Microbiol Infect 19, no. 10: 889-901.

Bouwman, AS, SL Kennedy, R Muller, RH Stephens, M Holst, AC Caffell, CA Roberts, and TA Brown. 2012. Genotype of a historic strain of Mycobacterium tuberculosis. Proc Natl Acad Sci U

344 S A 109, no. 45: 18511-18516.

345 Brosch, R, SV Gordon, M Marmiesse, P Brodin, C Buchrieser, K Eiglmeier, T Garnier, C

346 Gutierrez, G Hewinson, K Kremer, LM Parsons, AS Pym, S Samper, D van Soolingen, and ST

347 Cole. 2002. A new evolutionary scenario for the Mycobacterium tuberculosis complex. Proc Natl

348 Acad Sci US A 99, no. 6: 3684-3689.

Chan, JZ, MJ Sergeant, OY Lee, DE Minnikin, GS Besra, I Pap, M Spigelman, HD Donoghue, no. 3: 289-290.

Cole, ST, R Brosch, J Parkhill, T Garnier, C Churcher, D Harris, SV Gordon, K Eiglmeier, S Gas, CE 3rd Barry, F Tekaia, K Badcock, D Basham, D Brown, T Chillingworth, R Connor, R Davies, K Devlin, T Feltwell, S Gentles, N Hamlin, S Holroyd, T Hornsby, K Jagels, A Krogh, J McLean, S Moule, L Murphy, K Oliver, J Osborne, MA Quail, MA Rajandream, J Rogers, S Rutter, K Seeger, J Skelton, R Squares, S Squares, JE Sulston, K Taylor, S Whitehead, and BG Barrell. 1998. Deciphering the biology of Mycobacterium tuberculosis from the complete genome sequence. Nature 393, no. 6685: 537-544.

362 Colebunders, R, and I Bastian. 2000. A review of the diagnosis and treatment of smear-negative pulmonary tuberculosis. Int J Tuberc Lung Dis 4, no. 2: 97-107.

364 Coll, F, M Preston, JA Guerra-Assuncao, G Hill-Cawthorn, D Harris, J Perdigao, M Viveiros, I 365 Portugal, F Drobniewski, S Gagneux, JR Glynn, A Pain, J Parkhill, R McNerney, N Martin, and 366 TG Clark. 2014. PolyTB: a genomic variation map for Mycobacterium tuberculosis. Tuberculosis 367 (Edinb) 94, no. 3: 346-354.

368 Comas, I, J Chakravartti, PM Small, J Galagan, S Niemann, K Kremer, JD Ernst, and S Gagneux. 369 2010. Human T cell epitopes of Mycobacterium tuberculosis are evolutionarily hyperconserved.

370 Nat Genet 42, no. 6: 498-503.

371 Cubillos-Ruiz, A, A Sandoval, V Ritacco, B Lopez, J Robledo, N Correa, I Hernandez-Neuta, 372 MM Zambrano, and P Del Portillo. 2010. Genomic signatures of the haarlem lineage of 
373

Mycobacterium tuberculosis: implications of strain genetic variation in drug and vaccine development. J Clin Microbiol 48, no. 10: 3614-3623.

de Jong, BC, I Adetifa, B Walther, PC Hill, M Antonio, M Ota, and RA Adegbola. 2010a. Differences between tuberculosis cases infected with Mycobacterium africanum, West African type 2, relative to Euro-American Mycobacterium tuberculosis: an update. FEMS Immunol Med Microbiol 58, no. 1: 102-105.

de Jong, BC, M Antonio, T Awine, K Ogungbemi, YP de Jong, S Gagneux, K DeRiemer, T Zozio, N Rastogi, M Borgdorff, PC Hill, and RA Adegbola. 2009. Use of spoligotyping and large sequence polymorphisms to study the population structure of the Mycobacterium tuberculosis complex in a cohort study of consecutive smear-positive tuberculosis cases in The Gambia. $J$ Clin Microbiol 47, no. 4: 994-1001.

de Jong, BC, M Antonio, and S Gagneux. 2010b. Mycobacterium africanum--review of an important cause of human tuberculosis in West Africa. PLoS Negl Trop Dis 4, no. 9: e744.

Drobniewski, F, V Nikolayevskyy, Y Balabanova, D Bang, and D Papaventsis. 2012. Diagnosis of tuberculosis and drug resistance: what can new tools bring us? Int J Tuberc Lung Dis 16, no. 7: 860-870.

Ehrlich, P. 1882. Referate aus den Verein fur innere Medicin zu Berlin. Deutsche Medizinische Wochenschrift 9, 246-249.

Fabre, M, Y Hauck, C Soler, JL Koeck, J van Ingen, D van Soolingen, G Vergnaud, and C Pourcel. 2010. Molecular characteristics of "Mycobacterium canettii" the smooth Mycobacterium tuberculosis bacilli. Infect Genet Evol 10, no. 8: 1165-1173.

Feuerriegel, S, CU Koser, D Bau, S Rusch-Gerdes, DK Summers, JA Archer, MA Marti-Renom, and S Niemann. 2011. Impact of Fgd1 and ddn diversity in Mycobacterium tuberculosis complex on in vitro susceptibility to PA-824. Antimicrob Agents Chemother 55, no. 12: 5718-5722.

Feuerriegel, S, CU Koser, E Richter, and S Niemann. 2013. Mycobacterium canettii is intrinsically resistant to both pyrazinamide and pyrazinoic acid. J Antimicrob Chemother 68, no. 6: $1439-1440$.

Fischer, N, H Rohde, D Indenbirken, T Gunther, K Reumann, M Lutgehetmann, T Meyer, S Kluge, M Aepfelbacher, M Alawi, and A Grundhoff. 2014. Rapid metagenomic diagnostics for suspected outbreak of severe pneumonia. Emerg Infect Dis 20, no. 6: 1072-1075.

Gardy, JL, JC Johnston, SJ Ho Sui, VJ Cook, L Shah, E Brodkin, S Rempel, R Moore, Y Zhao, R Holt, R Varhol, I Birol, M Lem, MK Sharma, K Elwood, SJ Jones, FS Brinkman, RC Brunham, and P Tang. 2011. Whole-genome sequencing and social-network analysis of a tuberculosis outbreak. N Engl J Med 364, no. 8: 730-739.

Gonzalo-Asensio, J, W Malaga, A Pawlik, C Astarie-Dequeker, C Passemar, F Moreau, F Laval, M Daffe, C Martin, R Brosch, and C Guilhot. 2014. Evolutionary history of tuberculosis shaped by conserved mutations in the PhoPR virulence regulator. Proc Natl Acad Sci US A 
410 Gutierrez, MC, P Supply, and R Brosch. 2009. Pathogenomics of mycobacteria. Genome Dyn 6, $411 \quad 198-210$.

412 Helb, D, M Jones, E Story, C Boehme, E Wallace, K Ho, J Kop, MR Owens, R Rodgers, P 413 Banada, H Safi, R Blakemore, NT Lan, EC Jones-Lopez, M Levi, M Burday, I Ayakaka, RD 414 Mugerwa, B McMillan, E Winn-Deen, L Christel, P Dailey, MD Perkins, DH Persing, and D 415 Alland. 2010. Rapid detection of Mycobacterium tuberculosis and rifampin resistance by use of 416 on-demand, near-patient technology. J Clin Microbiol 48, no. 1: 229-237.

417 Hingley-Wilson, SM, R Casey, D Connell, S Bremang, JT Evans, PM Hawkey, GE Smith, A 418 Jepson, S Philip, OM Kon, and A Lalvani. 2013. Undetected multidrug-resistant tuberculosis 419 amplified by first-line therapy in mixed infection. Emerg Infect Dis 19, no. 7: 1138-1141.

Jagielski, T, J van Ingen, N Rastogi, J Dziadek, PK Mazur, and J Bielecki. 2014. Current methods in the molecular typing of Mycobacterium tuberculosis and other mycobacteria. Biomed Res Int 2014, 645802.

Kamerbeek, J, L Schouls, A Kolk, M van Agterveld, D van Soolingen, S Kuijper, A Bunschoten, H Molhuizen, R Shaw, M Goyal, and J van Embden. 1997. Simultaneous detection and strain differentiation of Mycobacterium tuberculosis for diagnosis and epidemiology. J Clin Microbiol 35, no. 4: 907-914.

Kay, GL, MJ Sergeant, V Giuffra, P Bandiera, M Milanese, B Bramanti, R Bianucci, and MJ Pallen. 2014. Recovery of a Medieval Brucella melitensis Genome Using Shotgun Metagenomics. MBio 5, no. 4:

430 Keller, A, A Graefen, M Ball, M Matzas, V Boisguerin, F Maixner, P Leidinger, C Backes, R 431 Khairat, M Forster, B Stade, A Franke, J Mayer, J Spangler, S McLaughlin, M Shah, C Lee, TT 432 Harkins, A Sartori, A Moreno-Estrada, B Henn, M Sikora, O Semino, J Chiaroni, S Rootsi, NM 433 Myres, VM Cabrera, PA Underhill, CD Bustamante, EE Vigl, M Samadelli, G Cipollini, J Haas, 434 H Katus, BD O'Connor, MR Carlson, B Meder, N Blin, E Meese, CM Pusch, and A Zink. 2012. 435 New insights into the Tyrolean Iceman's origin and phenotype as inferred by whole-genome 436 sequencing. Nat Commun 3, 698.

437 Kent, PT, and GP Kubica 1985. Public health mycobacteriology: a guide for the level III 438 laboratory. Centers for Disease Control and Prevention, Atlanta, Ga

439 Koboldt, DC, Q Zhang, DE Larson, D Shen, MD McLellan, L Lin, CA Miller, ER Mardis, L 440 Ding, and RK Wilson. 2012. VarScan 2: somatic mutation and copy number alteration discovery 441 in cancer by exome sequencing. Genome Res 22, no. 3: 568-576.

442 Koch, R. 1882. Die Aetiologie der Tubercukulose. Berliner Klinische Wochenschrift 19, 221-230.

443 Kohl, TA, R Diel, D Harmsen, J Rothganger, KM Walter, M Merker, T Weniger, and S Niemann. 444 2014. Whole-Genome-Based Mycobacterium tuberculosis Surveillance: a Standardized, Portable, 445 and Expandable Approach. J Clin Microbiol 52, no. 7: 2479-2486.

446 Köser, Claudio U., Matthew J. Ellington, and Sharon J. Peacock. 2014. Whole-genome 447 sequencing to control antimicrobial resistance. Trends in Genetics 
484 Nicol, MP, and RJ Wilkinson. 2008. The clinical consequences of strain diversity in
485 Mycobacterium tuberculosis. Trans R Soc Trop Med Hyg 102, no. 10: 955-965. 485

Koser, CU, JM Bryant, J Becq, ME Torok, MJ Ellington, MA Marti-Renom, AJ Carmichael, J Parkhill, GP Smith, and SJ Peacock. 2013. Whole-genome sequencing for rapid susceptibility testing of M. tuberculosis. N Engl J Med 369, no. 3: 290-292.

Koser, CU, MJ Ellington, EJ Cartwright, SH Gillespie, NM Brown, M Farrington, MT Holden, G Dougan, SD Bentley, J Parkhill, and SJ Peacock. 2012. Routine use of microbial whole genome sequencing in diagnostic and public health microbiology. PLoS Pathog 8, no. 8: e1002824.

Langmead, B, and SL Salzberg. 2012. Fast gapped-read alignment with Bowtie 2. Nat Methods 9, no. 4: 357-359.

Lanzas, F, PC Karakousis, JC Sacchettini, and TR Ioerger. 2013. Multidrug-resistant tuberculosis in panama is driven by clonal expansion of a multidrug-resistant Mycobacterium tuberculosis strain related to the KZN extensively drug-resistant $M$. tuberculosis strain from South Africa. $J$ Clin Microbiol 51, no. 10: 3277-3285.

Lim, YW, R Schmieder, M Haynes, D Willner, M Furlan, M Youle, K Abbott, R Edwards, J Evangelista, D Conrad, and F Rohwer. 2012. Metagenomics and metatranscriptomics: Windows on CF-associated viral and microbial communities. J Cyst Fibros

Loman, NJ, C Constantinidou, JZ Chan, M Halachev, M Sergeant, CW Penn, ER Robinson, and MJ Pallen. 2012a. High-throughput bacterial genome sequencing: an embarrassment of choice, a world of opportunity. Nat Rev Microbiol 10, no. 9: 599-606.

Loman, NJ, C Constantinidou, M Christner, H Rohde, JZ Chan, J Quick, JC Weir, C Quince, GP Smith, JR Betley, M Aepfelbacher, and MJ Pallen. 2013. A culture-independent sequence-based metagenomics approach to the investigation of an outbreak of Shiga-toxigenic Escherichia coli O104:H4. JAMA 309, no. 14: 1502-1510.

Loman, NJ, RV Misra, TJ Dallman, C Constantinidou, SE Gharbia, J Wain, and MJ Pallen. 2012b. Performance comparison of benchtop high-throughput sequencing platforms. Nat Biotechnol 30, no. 5: 434-439.

Lysholm, F, A Wetterbom, C Lindau, H Darban, A Bjerkner, K Fahlander, AM Lindberg, B Persson, T Allander, and B Andersson. 2012. Characterization of the viral microbiome in patients with severe lower respiratory tract infections, using metagenomic sequencing. PLoS One 7, no. 2: e30875.

Maiga, M, S Siddiqui, S Diallo, B Diarra, B Traore, YR Shea, AM Zelazny, BP Dembele, D Goita, H Kassambara, AS Hammond, MA Polis, and A Tounkara. 2012. Failure to recognize nontuberculous mycobacteria leads to misdiagnosis of chronic pulmonary tuberculosis. PLoS One 7, no. 5: e36902.

Matsen, FA, RB Kodner, and EV Armbrust. 2010. pplacer: linear time maximum-likelihood and Bayesian phylogenetic placement of sequences onto a fixed reference tree. BMC Bioinformatics 11,538 . 
Pallen, MJ. 2014. Diagnostic metagenomics: potential applications to bacterial, viral and parasitic infections. Parasitology 1-7.

Pfyffer, GE, HM Welscher, P Kissling, C Cieslak, MJ Casal, J Gutierrez, and S Rusch-Gerdes. 1997. Comparison of the Mycobacteria Growth Indicator Tube (MGIT) with radiometric and solid culture for recovery of acid-fast bacilli. J Clin Microbiol 35, no. 2: 364-368.

Roetzer, A, R Diel, TA Kohl, C Ruckert, U Nubel, J Blom, T Wirth, S Jaenicke, S Schuback, S Rusch-Gerdes, P Supply, J Kalinowski, and S Niemann. 2013. Whole genome sequencing versus traditional genotyping for investigation of a Mycobacterium tuberculosis outbreak: a longitudinal molecular epidemiological study. PLoS Med 10, no. 2: e1001387.

Schuenemann, VJ, P Singh, TA Mendum, B Krause-Kyora, G Jager, KI Bos, A Herbig, C Economou, A Benjak, P Busso, A Nebel, JL Boldsen, A Kjellstrom, H Wu, GR Stewart, GM Taylor, P Bauer, OY Lee, HH Wu, DE Minnikin, GS Besra, K Tucker, S Roffey, SO Sow, ST Cole, K Nieselt, and J Krause. 2013. Genome-wide comparison of medieval and modern Mycobacterium leprae. Science 341, no. 6142: 179-183.

Shamputa, IC, L Rigouts, LA Eyongeta, NA El Aila, A van Deun, AH Salim, E Willery, C Locht, P Supply, and F Portaels. 2004. Genotypic and phenotypic heterogeneity among Mycobacterium tuberculosis isolates from pulmonary tuberculosis patients. J Clin Microbiol 42, no. 12: 55285536 .

Shaw, JB, and N Wynn-Williams. 1954. Infectivity of pulmonary tuberculosis in relation to sputum status. American review of tuberculosis 69, no. 5: 724-732.

Stamatakis, A. 2014. RAxML version 8: a tool for phylogenetic analysis and post-analysis of large phylogenies. Bioinformatics 30, no. 9: 1312-1313.

Sweeney, FP, O Courtenay, A Ul-Hassan, V Hibberd, LA Reilly, and EM Wellington. 2006. Immunomagnetic recovery of Mycobacterium bovis from naturally infected environmental samples. Lett Appl Microbiol 43, no. 4: 364-369.

Walker, TM, CL Ip, RH Harrell, JT Evans, G Kapatai, MJ Dedicoat, DW Eyre, DJ Wilson, PM Hawkey, DW Crook, J Parkhill, D Harris, AS Walker, R Bowden, P Monk, EG Smith, and TE Peto. 2013. Whole-genome sequencing to delineate Mycobacterium tuberculosis outbreaks: a retrospective observational study. Lancet Infect Dis 13, no. 2: 137-146.

Walker, TM, MK Lalor, A Broda, L Saldana Ortega, M Morgan, L Parker, S Churchill, K Bennett, T Golubchik, AP Giess, C Del Ojo Elias, KJ Jeffery, IC Bowler, IF Laurenson, A Barrett, F Drobniewski, ND McCarthy, LF Anderson, I Abubakar, HL Thomas, P Monk, EG Smith, AS Walker, DW Crook, TE Peto, and CP Conlon. 2014. Assessment of Mycobacterium tuberculosis transmission in Oxfordshire, UK, 2007-12, with whole pathogen genome sequences: an observational study. Lancet Respir Med 2, no. 4: 285-292.

Wang, CS, HC Chen, IW Chong, JJ Hwang, and MS Huang. 2008. Predictors for identifying the most infectious pulmonary tuberculosis patient. J Formos Med Assoc 107, no. 1: 13-20. 
523 Wang, JY, HL Hsu, MC Yu, CY Chiang, FL Yu, CJ Yu, LN Lee, and PC Yang. 2011. Mixed 524 infection with Beijing and non-Beijing strains in pulmonary tuberculosis in Taiwan: prevalence, 525 risk factors, and dominant strain. Clin Microbiol Infect 17, no. 8: 1239-1245.

526 Warren, RM, TC Victor, EM Streicher, M Richardson, N Beyers, NC Gey van Pittius, and PD van 527 Helden. 2004. Patients with active tuberculosis often have different strains in the same sputum 528 specimen. Am J Respir Crit Care Med 169, no. 5: 610-614.

529 WHO. 2011. WHO Policy Xpert MTB/RIF Policy statement: automated real-time nucleic acid 530 amplification technology for rapid and simultaneous detection of tuberculosis and rifampicin 531 resistance: Xpert MTB/RIF system. WHO/HTM/TB/2011.4.,

532 WHO. 2013. Global tuberculosis report 2013. World Health Organization.

533 Wilson, MR, SN Naccache, E Samayoa, M Biagtan, H Bashir, G Yu, SM Salamat, S Somasekar, 534 S Federman, S Miller, R Sokolic, E Garabedian, F Candotti, RH Buckley, KD Reed, TL Meyer, 535 CM Seroogy, R Galloway, SL Henderson, JE Gern, JL DeRisi, and CY Chiu. 2014. Actionable 536 diagnosis of neuroleptospirosis by next-generation sequencing. $N$ Engl J Med 370, no. 25: 24085372417.

538 Zetola, NM, SS Shin, KA Tumedi, K Moeti, R Ncube, M Nicol, RG Collman, JD Klausner, and C 539 Modongo. 2014. Mixed Mycobacterium tuberculosis Complex Infections and False-Negative 540 Results for Rifampin Resistance by GeneXpert MTB/RIF Are Associated with Poor Clinical 541 Outcomes. J Clin Microbiol 52, no. 7: 2422-2429. 


\section{Figure 1}

Figure 1 Maximum likelihood tree tree showing placement of mycobacterial metagenome-derived genomes amongst the major lineages and clades within the $M$. tuberculosis complex.

Detection and characterisation of Mycobacterium tuberculosis in sputum samples using shotgun metagenomics Two representatives from each lineage/clades are shown. Tree calculated using RaXML and rooted with M. canetti (not shown)

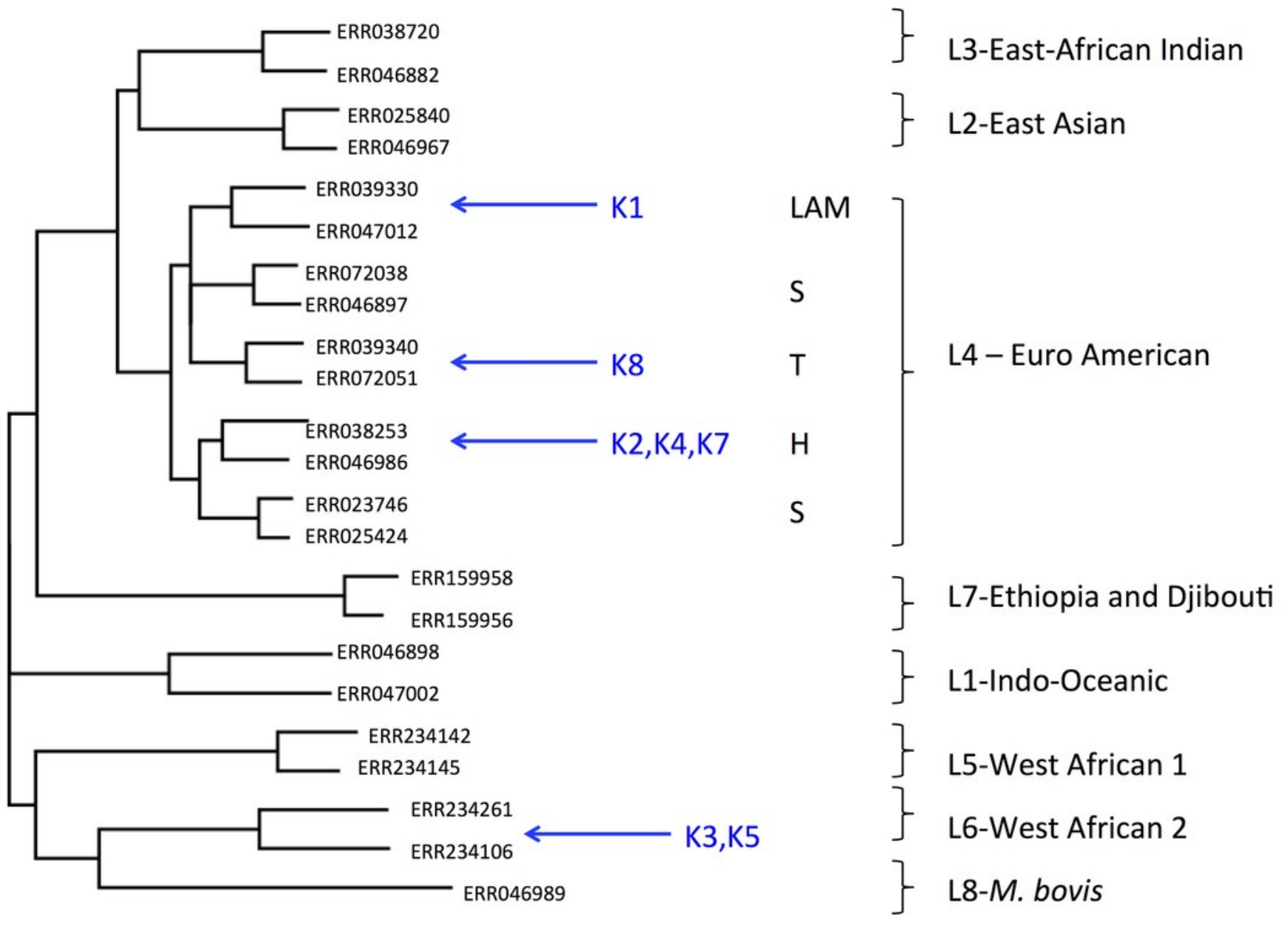

